Владикавказский математический журнал

2018, Том 20, Выпуск 4, С. 43-49

УДК 519.17

DOI 10.23671/VNC.2018.4.23386

\title{
ОБ АВТОМОРФИЗМАХ СИЛЬНО РЕГУЛЯРНОГО ГРАФА С ПАРАМЕТРАМИ $(117,36,15,9)$
}

\author{
А. К. Гутнова ${ }^{1}$, А. А. Махнев ${ }^{2}$ \\ ${ }^{1}$ Северо-Осетинский государственный университет им. К. Л. Хетагурова, \\ Россия, 362025, Владикавказ, ул. Ватутина, 44-46; \\ ${ }^{2}$ Институт математики и механики им. Н. Н. Красовского, \\ Россия, 620990, Екатеринбург, ул. С. Ковалевской, 16 \\ E-mail: gutnovaalina@gmail.com, makhnev@imm.uran.ru
}

\begin{abstract}
Аннотация. В предшествующих работах авторов найдены массивы пересечений дистанционно регулярных графов, в которых окрестности вершин являются псевдогеометрическими графамии для $p G_{s-3}(s, t)$. В частности, локально псевдо $p G_{2}(5,2)$-граф является сильно регулярным графом с параметрами $(117,36,15,9)$. Основным результатом данной статьи является теорема, в которой найдены возможные порядки и строение подграфов неподвижных точек автоморфизмов сильно регулярного графа с параметрами $(117,36,15,9)$. Этот граф имеет спектр $36^{1}, 9^{2} 6,-3^{9} 0$. Порядок клики в Г не превосходит $1+36 / 3=13$, порядок коклики в $\Gamma$ не превосходит $117 \cdot 3 / 39=9$. Далее из этого результата выведено следствие, что если группа $G$ автоморфизмов сильно регулярного графа с параметрами $(117,36,15,9)$ действует транзитивно на множестве вершин, то цоколь $T$ группы $G$ изоморфен либо $L_{3}(3)$ и $T_{a} \cong G L_{2}(3)$ - подгруппа индекса 117 , либо $T \cong L_{4}(3)$ и $T_{a} \cong U_{4}(2) . Z_{2}-$ подгруппа индекса 117.
\end{abstract}

Ключевые слова: сильно регулярный граф, симметричный граф, группа автоморфизмов графа. Mathematical Subject Classification (2010): 20D45.

Образец цитирования: Гутнова $A . K$., Махнев $A$. А. Об автоморфизмах сильно регулярного графа с параметрами $(117,36,15,9)$ // Владикавк. мат. журн.-2018.-T. 20, вып. 4.-С. 43-49. DOI: 10.23671/VNC.2018.4.23386.

\section{1. Введение}

Мы рассматриваем неориентированные графы без петель и кратных ребер. Для вершины $a$ графа $\Gamma$ через $\Gamma_{i}(a)$ обозначим $i$-окрестность вершины $a$, т. е. подграф, индуцированный $\Gamma$ на множестве всех вершин, находящихся на расстоянии $i$ от $a$. Положим $[a]=\Gamma_{1}(a), a^{\perp}=\{a\} \cup[a]$.

Степенъю вершины называется число вершин в ее окрестности. Граф $\Gamma$ называется регулярным степени $k$, если степень любой вершины из $\Gamma$ равна $k$. Граф $Г$ назовем реберно регулярным $с$ параметрами $(v, k, \lambda)$, если он содержит $v$ вершин, регулярен степени $k$, и каждое его ребро лежит в $\lambda$ треугольниках. Граф $Г-$ вполне регулярный граф c параметрами $(v, k, \lambda, \mu)$, если он реберно регулярен с соответствующими параметрами, и $[a] \cap[b]$ содержит $\mu$ вершин для любых двух вершин $a, b$, находящихся на расстоянии 2 в Г. Вполне регулярный граф диаметра 2 называется сильно регулярным графом.

(c) 2018 Гутнова А. К., Махнев А. А. 
В работах А. А. Махнева и А. К. Гутновой [1-3] найдены массивы пересечений дистанционно регулярных графов, в которых окрестности вершин являются псевдогеометрическими графамии для $p G_{s-3}(s, t)$. В частности, локально псевдо $p G_{2}(5,2)$-граф является сильно регулярным графом с параметрами $(117,36,15,9)$.

В данной работе найдены возможные автоморфизмы для сильно регулярного графа с параметрами $(117,36,15,9)$. Этот граф имеет спектр $36^{1}, 9^{26},-3^{90}$. Порядок клики в Г не превосходит $1+36 / 3=13$, порядок коклики в Г не превосходит $117 \cdot 3 / 39=9$.

\section{2. Вспомогательные результаты}

Лемма 1. Пусть Г - дистанционно регулярный граф с параметрами $(117,36,15,9)$, $G=\operatorname{Aut}(\Gamma)$. Если $g \in G, \chi_{1}-$ характер проекции представления $\psi$ на подпространство размерности 26, то $\alpha_{i}(g)=\alpha_{i}\left(g^{l}\right)$ для любого натурального числа $l$, взаимно простого $c|g|, \chi_{1}(g)=\left(\alpha_{0}(g)+\alpha_{1}(g) / 3-13\right) / 4$. Если $|g|=p-$ простое число, то $\chi_{1}(g)-26$ делится на $p$. Если $|g|=p^{2}, p-$ простое число, то $p^{2}$ делит $\chi_{1}\left(g^{p}\right)-26$.

$\triangleleft$ Имеем

$$
Q=\left(\begin{array}{ccc}
1 & 1 & 1 \\
26 & 13 / 2 & -13 / 4 \\
90 & -15 / 2 & 9 / 4
\end{array}\right)
$$

Поэтому $\chi_{1}(g)=\left(8 \alpha_{0}(g)+2 \alpha_{1}(g)-\alpha_{2}(g)\right) / 36$. Подставляя $\alpha_{2}(g)=117-\alpha_{0}(g)-\alpha_{1}(g)$, получим $\chi_{1}(g)=\left(\alpha_{0}(g)+\alpha_{1}(g) / 3-13\right) / 4$.

Остальные утверждения леммы следуют из [5, лемма 1$].$

Лемма 2. Пусть Г - дистанционно регулярный граф с параметрами $(117,36,15,9)$, $A-$ трехвершинный подграф из $\Gamma, y_{i}-$ число вершин из $\Gamma-A$, смежных точно $c i$ вершинами из $A$. Если $A-$ коклика, то $y_{0}=33-y_{3}$, если $A-$ объединение изолированной вершины и ребра, то $y_{0}=40-y_{3}$, если $A-$ геодезический путь, то $y_{0}=47-y_{3}$, а если $A-$ клика, то $y_{0}=54-y_{3}$.

$\triangleleft$ Пусть $A-$ коклика. Тогда $y_{1}=54+3 y_{3}, y_{2}=27-3 y_{3}$ и $y_{0}=33-3 y_{3}$. Пусть $A-$ клика. Тогда $y_{1}=18+3 y_{3}, y_{2}=42-3 y_{3}$ и $y_{0}=54-3 y_{3}$. Аналогично рассматриваются оставшиеся случаи. $\triangleright$

Пусть до конца работы $Г$ - сильно регулярный граф с параметрами $(117,36,15,9)$, $G=\operatorname{Aut}(\Gamma), g-$ элемент простого порядка $p$ из $G$ и $\Omega=\operatorname{Fix}(g)$. Заметим, что если $a$, $b$ - две вершины из $\Omega$ и $p>13$, то $[a] \cap[b] \subset \Omega$.

Лемма 3. Выполняются следующие утверждения:

(1) в Г нет собственных сильно регулярных подграфов с параметрами $\left(v^{\prime}, k^{\prime}, 15,9\right)$;

(2) если $\Omega-$ пустой граф, то $p=13, \alpha_{1}(g)=39$ и $\alpha_{2}(g)=78$ или $p=3, \alpha_{1}(g)=36 l-9$ и $\alpha_{2}(g)=126-36 l$;

(3) если $\Omega$ является $n$-кликой, то $n>1$ и либо $p=5, n=2,7,12, \alpha_{1}(g)=60 l+75-15 n$ и $\alpha_{2}(g)=42+14 n-60 l$, либо $p=2, n=5,7,9,11,13, \alpha_{1}(g)=24 l+39-3 n$ и $\alpha_{2}(g)=$ $78+2 n-24 l ;$

(4) если $\Omega$ не является кликой или пустым графом, то $\Omega$ содержит геодезический 2 -путь и $p \leqslant 13$.

$\triangleleft$ Пусть $\Delta-$ сильно регулярный граф с параметрами $\left(v^{\prime}, k^{\prime}, 15,9\right)$. Так как $n^{2}=$ $36+4\left(k^{\prime}-9\right)$, то $n=2 u, k^{\prime}=u^{2}$ и $\Delta$ имеет собственные значения $u+3,-(u-3)$. Кратность $u+3$ равна $(u-4) u\left(u^{2}+u-3\right) / 18$, поэтому $u \geqslant 6$.

Пусть $\Omega$ - пустой граф. Так как $117=13 \cdot 9$, то $p \in\{3,13\}$. Положим $\alpha_{i}(g)=p w_{i}$. Пусть $p=13$. Тогда $\chi_{1}(g)=13\left(w_{1} / 3-1\right) / 4$, поэтому $\alpha_{1}(g)=39$ и $\alpha_{2}(g)=78$. Пусть 
$p=3$. Тогда $\chi_{1}(g)=\left(w_{1}-13\right) / 4$ сравнимо с 2 по модулю 3 , поэтому $\alpha_{1}(g)=36 l-9$ и $\alpha_{2}(g)=126-36 l$.

Пусть $\Omega$ является $n$-кликой, $a-$ вершина из $\Omega$. Если $n=1$, то $p$ делит 36 и 80 , поэтому $p=2$, противоречие с тем, что для вершины $u \in \Gamma-a^{\perp}$, подграф $[u] \cap\left[u^{g}\right]$ пересекает $\Omega$.

Если $n>1$, то $p$ делит 20,50 и $17-n$, поэтому либо $p=5$ и $n=2,7,12$, либо $p=2$ и $n=3,5, \ldots, 13$. В любом случае $\chi_{1}(g)=\left(n+p w_{1} / 3-13\right) / 4$.

В случае $p=5$ имеем $\alpha_{1}(g)=60 l+75-15 n$ и $\alpha_{2}(g)=42+14 n-60 l$. В случае $p=2$ число $\left(n+2 w_{1} / 3-13\right) / 4$ четно, поэтому $\alpha_{1}(g)=24 l+39-3 n$ и $\alpha_{2}(g)=78+2 n-24 l$. Если $n=3$, то некоторая вершина из $\Gamma-\Omega$ не смежна с вершинами из $\Omega$, противоречие.

Пусть $\Omega$ является $m$-кликой, $m>1$. Тогда $p$ делит 9 и 26 , противоречие.

Пусть $\Omega$ содержит ребро и является обьединением $t \geqslant 2$ изолированных клик. Тогда $p$ делит 9 и 20 , противоречие.

Пусть $\Omega$ содержит геодезический 2-путь. Если $p>13$, то $\Omega-$ сильно регулярный граф с $\lambda=15$ и $\mu=9$, противоречие с утверждением (1). $\triangleright$

Лемма 4. Пусть $\Omega$ содержит геодезический путь $b, a, c$. Тогда выполняются следующие утверждения:

(1) если $\Omega$ содержит вершину а степени 36 , то либо $p=3$ и $\alpha_{0}(g)=45$, либо $p=2$, $37 \leqslant \alpha_{0}(g) \leqslant 63$ и число $\alpha_{0}(g)+\alpha_{1}(g) / 3-13$ делится на $8 ;$

(2) $p$ не больше 7 ;

(3) если $p=7$, то $\Omega-$ сильно регулярный граф с параметрами $(26,15,8,9)$ и $\alpha_{1}(g)=24$.

$\triangleleft$ Пусть $\Omega$ содержит вершину а степени 36 . Ввиду леммы 2 любая $\langle g\rangle$-орбита длины $p$ не содержит 3 -коклик. Так как любая вершина из $\Gamma-a^{\perp}$ смежна с 9 вершинами из $[a]$, то любая $\langle g\rangle$-орбита длины $p$ не содержит геодезических 2-путей. Если $p>2$, то любая $\langle g\rangle$-орбита длины $p$ является кликой и $p \leqslant 7$. В этом случае

$$
\chi_{1}(g)=\left(\alpha_{0}(g)+\left(117-\alpha_{0}(g)\right) / 3-13\right) / 4,
$$

поэтому $\alpha_{0}(g)=6 l+3$ для $p>3$ и $\alpha_{0}(g)=18 l+9$ для $p=3$. Отсюда $p=3$ и $\alpha_{0}(g)=45$.

Если $p=2$, то число $\chi_{1}(g)=\left(\alpha_{0}(g)+\alpha_{1}(g) / 3-13\right) / 4$ четно. Утверждение (1) доказано.

Пусть $p=13$. Тогда $\lambda_{\Omega}=2,15, \mu_{\Omega}=9,|\Omega|=13,26,39,52$ и степени вершин в $\Omega$ равны 10,23 . Если $|\Omega|=13$, то $\Omega$ - сильно регулярный граф с параметрами $(13,10,2,9)$, противоречие.

Пусть $|\Omega|=26$. Если $\Omega$ содержит вершину $а$ степени 23 , то число ребер между $\Omega(a)$ и $\Omega_{2}(a)$ равно 18, но не меньше $23 \cdot 7$, противоречие. Значит, $\Omega$ - сильно регулярный граф с параметрами $(26,10,2,9)$, противоречие.

Пусть $|\Omega|=39$. Если $\Omega$ содержит вершину а степени 10 , то число ребер между $\Omega(a)$ и $\Omega_{2}(a)$ равно $28 \cdot 9$, но не больше $20 \cdot 10$, противоречие. Значит, $\Omega-$ регулярный граф степени 23 , противоречие.

Пусть $|\Omega|=52$. Если $\Omega$ содержит вершину $а$ степени 10 , то число ребер между $\Omega(a)$ и $\Omega_{2}(a)$ равно $41 \cdot 9$, но не больше $10 \cdot 21$, противоречие. Значит, $\Omega-$ регулярный граф степени 23 , и число ребер между $\Omega(a)$ и $\Omega_{2}(a)$ равно $28 \cdot 9=20 y+7(23-y)$, поэтому $y=13$. Но если $b, c-$ две вершины степени 2 в графе $\Omega(a)$, то $[b] \cap[c]$ содержит 13 вершин из $[a]-\Omega$ и не менее 12 вершин из $\Omega_{2}(a)$, противоречие.

Пусть $p=11$. Тогда $\lambda_{\Omega}=4,15, \mu_{\Omega}=9,|\Omega|=18,29,40,51$ и степени вершин в $\Omega$ равны 14,25 . Если $\Omega$ содержит вершину а степени 14 , то число ребер между $\Omega(a)$ и $\Omega_{2}(a)$ не меньше $14 \cdot 9$, равно $9(|\Omega|-15)$, но не больше $14 \cdot 20$, поэтому $|\Omega|=40$ и указанное число 
равно $9 \cdot 25=20 y+9(14-y) \cdot y=9$. Но если $b, c-$ две вершины из $\Omega(a)$, смежные с 20 вершинами из $\Omega_{2}(a)$, то $[b] \cap[c]$ содержит $a$ и не менее 15 вершин из $\Omega_{2}(a)$, противоречие.

Значит, $\Omega$ - регулярный граф степени $25,|\Omega|=40$ и число ребер между $\Omega(a)$ и $\Omega_{2}(a)$ равно $14 \cdot 9$, но не меньше $9 \cdot 25$, противоречие.

Пусть $p=7$. Тогда $\lambda_{\Omega}=1,8,15, \mu_{\Omega}=2,9,|\Omega|=19,26,33,40,47,54$ и степени вершин в $\Omega$ равны $8,15,22,29$. Если $|\Omega|>33$, то любая $\langle g\rangle$-орбита длины 7 не содержит 3 -коклик. Далее, $\chi_{1}(g)=\left(\alpha_{0}(g)+\alpha_{1}(g) / 3-13\right) / 4$ и в случае $\alpha_{0}(g)=40$ имеем $\alpha_{1}(g)=63$. В этом случае на $\Gamma-\Omega$ имеется 5 кликовых $\langle g\rangle$-орбит и 6 орбит степени 4 . Противоречие с тем, что для ребра и изолированной от него вершины из $\langle g\rangle$-орбиты степени 4 , подграф, состоящий из вершин, смежных с 0 или 3 вершинами из этой тройки, содержит 40 вершин из $\Omega$ и 2 вершины из этой $\langle g\rangle$-орбиты, противоречие. В случае $\alpha_{0}(g)=47$ имеем $\alpha_{1}(g)=42$. В этом случае для геодезического 2 -пути из $\langle g\rangle$-орбиты подграф, состоящий из вершин, смежных с 0 или 3 вершинами из этой тройки, содержит 47 вершин из $\Omega$ и 2 вершины из этой $\langle g\rangle$-орбиты, противоречие. В случае $\alpha_{0}(g)=54$ имеем $\alpha_{1}(g)=63$ и $\chi_{1}(g)=(41+21) / 4$, противоречие.

Значит, $|\Omega| \leqslant 33$. Если $\Omega$ содержит вершину а степени 8 , то число ребер между $\Omega(a)$ и $\Omega_{2}(a)$ не меньше $8 \cdot 6$ и равно $2(|\Omega|-9)$, поэтому $|\Omega|=33$ и $\Omega$ - сильно регулярный граф с параметрами $(33,8,1,2)$. В этом случае $\Omega$ имеет собственные значения $2,-3$ и кратность 2 равна $2 \cdot 8 \cdot 11 / 10$, противоречие.

Если $\Omega$ содержит вершину $а$ степени 22 , то число ребер между $\Omega(a)$ и $\Omega_{2}(a)$ не меньше $22 \cdot 6$ и не больше $10 \cdot 9$, противоречие. Значит, $\Omega$ - регулярный граф степени $15,|\Omega|=26$ и число ребер между $\Omega(a)$ и $\Omega_{2}(a)$ равно $15 \cdot 6=10 \cdot 9$. Отсюда $\Omega-$ сильно регулярный граф с параметрами $(26,15,8,9)$ и $\chi_{1}(g)=\left(13+\alpha_{1}(g) / 3\right) / 4$, поэтому $\alpha_{1}(g)=24$. $\triangleright$

Лемма 5. Пусть $\Omega$ содержит геодезический путь $b, a, c$. Тогда выполняются следующие утверждения:

(1) число $p$ не равно 5;

(2) если $p=3$, то $|\Omega| \leqslant 33$ или $|\Omega|=45$;

(3) если $p=2$, то $|\Omega| \leqslant 63$.

$\triangleleft$ Пусть $p=5$. Тогда $\lambda_{\Omega}=0,5,10,15, \mu_{\Omega}=4,9,|\Omega|=12,17,22,27,32,37,42,47,52$, степени вершин в $\Omega$ равны $6,11,16,21,26,31$ и $\chi_{1}(g)=\left(\alpha_{0}(g)+\alpha_{1}(g) / 3-13\right) / 4$.

Пусть $Y$ - множество вершин из $\Omega_{2}(a)$, смежных с 9 вершинами из $\Omega(a), y=|Y|$. Тогда число ребер между $\Omega(a)$ и $\Omega_{2}(a)$ равно $6|\Omega(a)|+5 x$. С другой стороны, указанное число ребер равно $9 y+4\left(\left|\Omega_{2}(a)\right|-y\right)$ и делится на 5 , противоречие.

Пусть $p=3$. Тогда $\lambda_{\Omega}=0,3, \ldots, 15, \mu_{\Omega}=0,3,6,9,|\Omega|=6,9, \ldots, 54$ и степени вершин в $\Omega$ равны $0,3,6, \ldots, 36$ и $\chi_{1}(g)=\left(\alpha_{0}(g)+\alpha_{1}(g) / 3-13\right) / 4$, поэтому $\left(\alpha_{0}(g)+\alpha_{1}(g) / 3-13\right) / 4$ сравнимо с 2 по модулю 3.

Если $|\Omega|>33$, то ввиду леммы 2 любая $\langle g\rangle$-орбита длины 3 является кликой, $\alpha_{1}(g)=117-\alpha_{0}(g)$ и $\left(2 \alpha_{0}(g) / 3+26\right) / 4$ сравнимо с 2 по модулю 3 . Поэтому $\alpha_{0}(g)=45$ и $\alpha_{1}(g)=72$.

Пусть $p=2$. Тогда $\lambda_{\Omega}=1,3, \ldots, 15, \mu_{\Omega}=1,3, \ldots, 9,|\Omega|=5,7, \ldots, 65$ и степени вершин в $\Omega$ равны $0,2,4, \ldots, 36$ и $\chi_{1}(g)=\left(\alpha_{0}(g)+\alpha_{1}(g) / 3-13\right) / 4$, поэтому $\left(\alpha_{0}(g)+\right.$ $\left.\alpha_{1}(g) / 3-13\right) / 4$ четно.

Если $|\Omega|>63$, то любая $\langle g\rangle$-орбита длины 2 является кликой, $\alpha_{1}(g)=117-\alpha_{0}(g)=52$ и $\chi_{1}(g)=(65+52 / 3-13) / 4$, противоречие. $\triangleright$

Лемма 6. Пусть Г является сильно регулярным графом с параметрами $(117,36,15,9)$ и группа $G=\operatorname{Aut}(\Gamma)$ действует транзитивно на множестве вершин графа Г. Пусть $f-$ элемент порядка 13 из $G, g$ - элемент простого порядка $p<13$ из $C_{G}(f)$. Тогда $\left|C_{G}(f)\right|$ 
делит 26 и если инволюция $g \in G$ централизует $f$, то $\operatorname{Fix}(g)$ является 13-кокликой и $\alpha_{1}(g)=0$.

$\triangleleft|G|$ делится на $9 \cdot 13$. По теореме $1 \pi(G) \subseteq\{2,3,5,7,13\}$.

Пусть $G$ содержит подгруппу $\langle h\rangle$ порядка $13 p, p$ - простое число, меньшее $11, g=h^{13}$, $f=h^{p}$. Ввиду теоремы $1 \operatorname{Fix}(f)$ - пустой граф, $p=2$ и либо $|\Omega|=13$ и $\alpha_{1}(g)=$ $24 l$ делится на 13 , либо $|\Omega|=39$. В последнем случае $\chi_{1}(g)=\left(\alpha_{1}(g) / 3+26\right) / 4$, число $\left(\alpha_{1}(g) / 3+26\right) / 4$ четно и $\alpha_{1}(g)=3(8 l-26)$ делится на 13 , противоречие. Из действия $g$ на $U_{i}=\left\{u \in \Gamma: d\left(u, u^{f^{i}}\right)=1\right\}$ следует, что $\Omega=\operatorname{Fix}(g)$ пересекает $U_{i}$ для любого $i$, не кратного 13 , поэтому $\Omega$ является 13 -кокликой.

Пусть $V$ - подгруппа порядка 4 из $C_{G}(f)$. Так как $\chi_{1}(g)-26$ не делится на 4 , то $V-$ элементарная абелева группа. Из действия $V$ на $U=\left\{u \in \Gamma: d\left(u, u^{f}\right)=1\right\}$ следует, что $\Omega=\operatorname{Fix}(g)$ содержится в $U$ для любой инволюции $g \in V$, противоречие с действием $V$ на $W=\left\{w \in \Gamma: d\left(w, w^{f}\right)=2\right\}$. $\triangleright$

\section{3. Основной результат}

Теорема 1. Пусть $\Gamma-$ сильно регулярный граф с параметрами $(117,36,15,9)$, $G=\operatorname{Aut}(\Gamma), g-$ элемент из $G$ простого порядка $p$ и $\Omega=\operatorname{Fix}(g)$. Тогда $\pi(G) \subseteq$ $\{2,3,5,7,13\}$ и выполняется одно из следующих утверждений:

(1) $\Omega$ - пустой граф, $p=13, \alpha_{1}(g)=39$ и $\alpha_{2}(g)=78$ или $p=3, \alpha_{1}(g)=36 l-9$ и $\alpha_{2}(g)=126-36 l$

(2) $\Omega$ является $n$-кликой, и либо $p=5, n=2,7,12, \alpha_{1}(g)=60 l+75-15 n$ и $\alpha_{2}(g)=$ $42+14 n-60 l$, либо $p=2, n=5,7,9,11,13, \alpha_{1}(g)=24 l+39-3 n$ и $\alpha_{2}(g)=78+2 n-24 l$;

(3) $\Omega$ содержит геодезический 2-путь и либо

(i) $p=7, \Omega$ - сильно регулярный граф с параметрами $(26,15,8,9)$ и $\alpha_{1}(g)=24$, либо

(ii) $p=3,|\Omega| \leqslant 33$ или $|\Omega|=45$, либо

(iii) $p=2$ и $|\Omega| \leqslant 63$.

$\triangleleft$ Доказательство теоремы опирается на метод Хигмена работы с автоморфизмами дистанционно регулярного графа, представленный в третьей главе монографии Камерона [4]. При этом граф Г рассматривается как симметричная схема отношений $(X, \mathscr{R})$ с $d$ классами, где $X-$ множество вершин графа, $R_{0}$ - отношение равенства на $X$ и для $i \leqslant 1$ класс $R_{i}$ состоит из пар $(u, w)$ таких, что $d(u, w)=i$. Для $u \in \Gamma$ положим $k_{i}=\left|\Gamma_{i}(u)\right|$, $v=|\Gamma|$. Классу $R_{i}$ отвечает граф $\Gamma_{i}$ на множестве вершин $X$, в котором вершины $u, w$ смежны, если $(u, w) \in R_{i}$. Пусть $A_{i}$ - матрица смежности графа $\Gamma_{i}$ для $i>0$ и $A_{0}=I-$ единичная матрица. Тогда $A_{i} A_{j}=\sum p_{i j}^{l} A_{l}$ для чисел пересечений $p_{i j}^{l}$.

Пусть $P_{i}$ - матрица, в которой на месте $(j, l)$ стоит $p_{i j}^{l}$. Тогда собственные значения $p_{1}(0), \ldots, p_{1}(d)$ матрицы $P_{1}$ являются собственными значениями графа $\Gamma$ кратностей $m_{0}=1, \ldots, m_{d}$. Матрицы $P$ и $Q$, у которых на месте $(i, j)$ стоят стоят $p_{j}(i)$ и $q_{j}(i)=m_{j} p_{i}(j) / k_{i}$ соответственно, называются первой и второй матрицей собственных значений схемы и связаны равенством $P Q=Q P=v I$.

Пусть $u_{j}$ и $w_{j}$ - левый и правый собственные векторы матрицы $P_{1}$, отвечающие собственному значению $p_{1}(j)$ и имеющие первую координату 1 . Тогда $w_{j}$ являются столбцами матрицы $P$ и $m_{j} u_{j}$ являются строками матрицы $Q$.

Подстановочное представление группы $G=\operatorname{Aut}(\Gamma)$ на вершинах графа $\Gamma$ обычным образом дает матричное представление $\psi$ группы $G$ в $G L(v, \mathbf{C})$. Пространство $\mathbf{C}^{v}$ является ортогональной прямой суммой собственных $G$-инвариантных подпространств 
$W_{0}, \ldots, W_{d}$ матрицы смежности $A=A_{1}$ графа $Г$. Для любого $g \in G$ матрица $\psi(g)$ перестановочна с $A$, поэтому подпространство $W_{i}$ является $\psi(G)$-инвариантным. Пусть $\chi_{i}-$ характер представления $\psi_{W_{i}}$. Тогда (см. $\left.[4, \S 3.7]\right)$ для $g \in G$ получим

$$
\chi_{i}(g)=v^{-1} \sum_{j=0}^{d} Q_{i j} \alpha_{j}(g),
$$

где $\alpha_{j}(g)$ - число точек $x$ из $X$ таких, что $d\left(x, x^{g}\right)=j$.

Утверждения 1)-2) следуют из леммы 3. Доказательство утверждения 3) следует из лемм $3-5 . \triangleright$

Следствие 1. Если группа $G$ автоморфизмов сильно регулярного графа с параметрами $(117,36,15,9)$ действует транзитивно на множестве вершин, то цоколь $T$ группы $G$ изоморфен либо $L_{3}(3)$ и $T_{a} \cong G L_{2}(3)$ - подгруппа индекса 117 , либо $T \cong L_{4}(3)$ и $T_{a} \cong U_{4}(2) \cdot Z_{2}$ - подгруппа индекса 117 .

$\triangleleft$ Ввиду леммы 6 имеем $S(G)=O_{3}(G)$. Пусть $\bar{G}=G / O_{3}(G), \bar{T}$ - цоколь группы $\bar{G}$. Из действия подгруппы порядка 13 на минимальной нормальной подгруппе $\bar{N}$ из $\bar{G}$ следует, что $|\bar{N}|$ делится на 13 . Отсюда $\bar{T}$ - простая неабелева группа и ввиду [6, таблица 1$]$ группа $\bar{T}$ изоморфна $L_{3}(3), L_{2}(25), U_{3}(4), P S p_{4}(5), L_{4}(3),{ }^{2} F_{4}(2)^{\prime}, L_{2}(13), L_{2}(27), G_{2}(3)$, ${ }^{3} D_{4}(2), S z(8), L_{2}(64), U_{4}(5), L_{3}(9), P S p_{6}(3), P \Omega_{7}(3), G_{2}(4), P S p_{4}(8), P \Omega_{8}^{+}(3)$.

Так как $\bar{T}$ содержит максимальную подгруппу индекса, делящего $13 \cdot 9$, то либо $\bar{T} \cong L_{3}(3)$ и $\bar{T}_{a} \cong G L_{2}(3)-$ подгруппа индекса 117 , либо $\bar{T} \cong L_{4}(3)$ и $\bar{T}_{a} \cong U_{4}(2) . Z_{2}-$ подгруппа индекса 117.

В любом случае $O_{3}(G)=1$. $\triangleright$

\section{Литература}

1. Гутнова A. K., Махнев A. А. Вполне регулярные графы, в которых окрестности вершин псевдогеометрические графы для $p G_{s-3}(s, t)$ // Докл. АН.-2014.-Т. 454, № 2.-С. 145-148. DOI: $10.7868 / \mathrm{S} 0869565214020042$.

2. Гутнова А. К., Махнев А. А. Локально псевдо $G Q(4, t)$-графы // Докл. АН.-2015.-T. 462, № 6.C. 637-641. DOI: $10.7868 / \mathrm{S} 086956521518005 \mathrm{X}$.

3. Гутнова A. K., Махнев А. А. Графы диаметра, не большего 3, в которых окрестности вершин псевдогеометрические графы для $p G_{s-3}(s, t) / /$ Докл. АН.-2015.-T. 461, № 6.-C. 629-632. DOI: 10.7868/S0869565215120038.

4. Cameron P. J. Permutation Groups.-Cambridge: Cambridge Univ. Press, 1999.-(London Math. Soc. Student Texts № 45).

5. Гаврилюк A. Л., Махнев А. А. Об автоморфизмах дистанционно регулярного графа с массивом пересечений $\{56,45,1 ; 1,9,56\} / /$ Докл. АН.-2010.-Т. 432, № 5.-С. 512-515.

6. Zavarnitsine A. V. Finite simple groups with narrow prime spectrum // Sibirean Electr. Math. Reports.-2009.-Vol. 6.-P. 1-12.

\section{Статъл поступила 29 мая 2018 г.}

Гутнова Алина КАзБековна

Северо-Осетинский государственный университет им. К. Л. Хетагурова,

доцент кафедры алгебры и геометрии

РОССИЯ, 362025, Владикавказ, ул. Ватутина, 44-46

E-mail: gutnovaalina@gmail.com

МАХнев АЛЕКСАНДР АЛЕКСЕЕВИч

Институт математики и механики им. Н. Н. Красовского, зав. отделом алгебры и топологии

РОССИЯ, 620990, Екатеринбург, ул. С. Ковалевской, 16

E-mail: makhnev@imm.uran.ru 


\title{
ON AUTOMORPHISMS OF A STRONGLY REGULAR GRAPH WITH PARAMETERS $(117,36,15,9)$
}

\author{
Gutnova, A. K. ${ }^{1}$ and Makhnev, A. A. ${ }^{2}$ \\ ${ }^{1}$ North Ossetian State University, \\ 44-46 Vatutin Street, Vladikavkaz 362025, Russia; \\ ${ }^{2}$ N. N. Krasovskii Institute of Mathematics and Mechanics, \\ 16 S. Kovalevskaja st., Ekaterinburg 620990, Russia \\ E-mail: gutnovaalina@gmail.com, makhnev@imm.uran.ru
}

\begin{abstract}
In the previous work of the authors some arrays of intersections of distance-regular graphs were found, in which the neighborhoods of the vertices are pseudogeometric graphs for $p G_{s-3}(s, t)$. In particular, a locally pseudo $p G_{2}(5,2)$-graph is a strongly regular graph with parameters $(117,36,15,9)$. The main result of this paper gives a description of possible orders and the structure of the subgraphs of fixed points of automorphisms of a strongly regular graph with parameters $(117,36,15,9)$. This graph has a spectrum of $36^{1}, 9^{2} 6,-3^{9} 0$. The order of clicks in $\Gamma$ does not exceed $1+36 / 3=13$, the order of the cocliques in $\Gamma$ does not exceed $117 \cdot 3 / 39=9$. Further, from this result, the following corollary is derived: if the group $\Gamma$ of automorphisms of a strongly regular graph with parameters $(117,36,15,9)$ acts transitively on the set of vertices, then the socle $T$ of the group $\Gamma$ is isomorphic to either $L_{3}(3)$ and $T_{a} \cong G L_{2}(3)$ is a subgroup of index 117 , or $T_{a} \cong G L_{2}(3)$ and $T_{a} \cong U_{4}(2) . Z_{2}$ is a subgroup of index 117 .
\end{abstract}

Key words: strongly regular graph, symmetric graph, automorphism groups of a graph.

Mathematical Subject Classification (2010): 20D45.

For citation: Gutnova, A. K. and Makhnev, A. A. On Automorphisms of a Strongly Regular Graph with Parameters $(117,36,15,9)$, Vladikavkaz Math. J., 2018, vol. 20, no. 4, pp. 43-49 (in Russian). DOI: 10.23671/VNC.2018.4.23386.

\section{References}

1. Gutnova, A. K. and Makhnev, A. A. Completely Regular Graphs in Which Neighborhoods of Vertices are Pseudogeometric Graphs for $p G_{s-3}(s, t)$, Doklady Mathematics, 2014, vol. 454, no. 2, pp. 145-148 (in Russian). DOI: 10.7868/S0869565214020042.

2. Gutnova, A. K. and Makhnev, A. A. Locally Pseudo GQ(4,t)-Graphs, Doklady Mathematics, 2015, vol. 462, no. 6, pp. 637-641 (in Russian). DOI: 10.7868/S086956521518005X.

3. Gutnova, A. K. and Makhnev, A. A. Graphs of Diameter not Greater than 3, in Which Neighborhoods of Vertices are Pseudogeometric Graphs for $p G_{s-3}(s, t)$, Doklady Mathematics, 2015, vol. 461, no. 6, pp. 629-632 (in Russian). DOI: 10.7868/S0869565215120038.

4. Cameron, P. J. Permutation Groups. London Math. Soc. Student Texts, no. 45, Cambridge, Cambridge Univ. Press, 1999.

5. Gavrilyuk, A. L. and Makhnev, A. A. On Automorphisms of Amply Regular Graphs with the Intersection Array $\{56,45,1 ; 1,9,56\}$, Doklady Mathematics, vol. 432, no. 5, pp. 512-515. (in Russian).

6. Zavarnitsine, A. V. Finite Simple Groups with Narrow Prime Spectrum, Sibirean Electr. Math. Reports, 2009, vol. 6, pp. 1-12.

Received May 29, 2018

Alina K. Gutnova

North Ossetian State University,

44-46 Vatutin Street, Vladikavkaz 362025, Russia,

Associate Professor of the Department of Algebra and Geometry

E-mail: gutnovaalina@gmail.com

Alexander A. Makhnev

N. N. Krasovskii Institute of Mathematics and Mechanics,

16 S. Kovalevskaja st., Ekaterinburg 620990, Russia,

Head of Departament of Algebra and Topology

E-mail: makhnev@imm.uran.ru

https://orcid.org/0000-0003-2868-6713 